\title{
Microstructural description of packed bed using Voronoi polyhedra
}

\author{
Y.C. Liao ${ }^{a}$, D.J. Lee ${ }^{\mathrm{a}, *}$, Pinjing He ${ }^{\mathrm{b}}$ \\ a Department of Chemical Engineering, National Taiwan University, Taipei 10617, Taiwan \\ ${ }^{\mathrm{b}}$ State Key Laboratory of Pollution Control and Resource Reuse, School of Environment Science and Engineering, Tongji University, \\ Shanghai 200092, China
}

Received 11 September 2000; received in revised form 11 December 2000; accepted 22 March 2001

\begin{abstract}
This work has examined the feasibility of using Voronoi polyhedron (VP) in describing the microstructure of a simulated packed bed with controlled disorderness. A transition in microstructure among the regularly packed (crystal-like) and randomly packed (liquid-like) states was detected over a wide range of the critical contact angles among particles. The mean asphericity and the entropy function of VP volume were noted as adequate indices for parking characteristics of the packed bed. (C) 2002 Elsevier Science B.V. All rights reserved.
\end{abstract}

Keywords: Voronoi polyhedron; Packed bed; Disorderness; Transition

\section{Introduction}

Voronoi polyhedra (VP), defined as the convex region of the close space to their own central particle, tessellate and fill the space of the multiparticle system [1]. The Voronoi-Delaunay tessellation was introduced to analyze random close packing of hard sphere [2-5] and has found applications in a variety of fields for identifying the microstructures of multiobject system [6-22].

The analysis of VP is especially effective in examining the local structure of a multiparticle system. The distributions of volume, surface area, numbers of faces, and asphericity parameter $(\eta)$ of VP occupied by constituting particles are proposed as a better microstructural descriptor than is the radial distribution function (RDF), since the latter has averaged out the structural information along the radial direction. The $\eta$ value is defined as $A^{3} / 36 \pi V^{2}$, where $A$ and $V$ are the surface area and volume of VP, respectively, and $\eta$ is unity for a sphere and is greater than unity for other shapes (for example, body-centered cubic, 1.33; octahedron, 1.65; simple cubic, 1.91; and tetrahedron, 3.31). Owing to the capability to identify the shape of the VP, many investigators recommended the use of $\eta$, instead of the other VP descriptors, in the differentiation of the detailed structures among phases [7,9,11]. Everett [23]

\footnotetext{
${ }^{*}$ Corresponding author. Tel.: +886-2-2362-5632; fax: $+886-2-2362-$ 3040.

E-mail address: djlee@ccms.ntu.edu.tw (D.J. Lee).
}

estimated the configurational entropy changes based on the face distribution of VP. Hsu and Mou [8] employed the same idea for estimating entropy change during melting an LJ solid.

Liao and Lee [24] investigated the feasibility of using VP to detect the existence of 'channel' (particle-free regime) in the multiparticle systems. They concluded that VP is not capable of detecting the existence of a channel region for a largely perturbed lattice system. Later, the same authors [25] discussed the general ability of VP to describe the configurations of a multiparticle system with a controlled disorderliness for three lattice systems: a freecentered cubic (fcc) lattice, body-centered cubic (bcc) lattice, and simple cubic (sc) lattice. Unlike most previous studies, these authors demonstrated that no single VP descriptor is better than the other ones for detecting the microstructural changes after perturbation to particles. They also proposed that all VP descriptors should be considered so as to provide a more comprehensive description to the microstructure of the multiparticle systems.

Detailed descriptions of microstructures in a packed bed are essential to predict its associated effective transport properties [26]. A packed bed is normally composed of hard particles. Restated, a particle has to physically come into contact with the other three particles below it to reach mechanical balance. The total volume of the packed bed of a fixed number of particles would change according to the way the particles are packed (variable-volume system). Tassopoulos and Rosner [27] discussed the employment of microstructural descriptors, such as volume fraction, mean 
and distribution of coordination number, contact normal distribution, and fabric tensor, on the description of their simulated packed bed. Spedding and Spencer [28] employed Voronoi polyhedron analysis to provide a much more precise measurement of the volume occupied by constituent particles in their simulated packed bed. However, no comprehensive work had been devoted to examine the feasibility of using VP analysis to describe the microstructure of a packed bed with well-controlled disorderliness.

In this work, we first generated a three-dimensional simulated packed bed with well-controlled disorderliness. Then, the VP analysis was adopted for characterizing the microstructures in the packed bed. The occurrence of 'phase transition' among the regularly packed (crystal-like) and randomly packed (liquid-like) state denotes a sudden change in the effective transport properties like thermal conductivity of the packed bed. We demonstrated that the VP are more sensitive than RDF to detect such a structural change.

\section{Methods}

We refined the two-dimensional rain model proposed by Ref. [29] to a three-dimensional version for making the packed bed. A parameter, termed as the critical angle $\theta_{c}$ [30], is adopted to represent the particle-particle interactions. A large $\theta_{c}$ denotes a strong particle-particle interaction. Particles were allowed to fall down from the top end of a rectangular parallelepiped cell with size $20(\mathrm{~L}) \times 20$ $(\mathrm{W}) \times 100(\mathrm{H})$ of particle's radius (Fig. 1a). Periodical boundary conditions were applied along the $x$ and the $y$ directions to simulate a very large particle system.

At the start of the simulation, a particle was placed at the top of the cell by randomly choosing its position from the lattice of $20 \times 20$ even grids. The particle's position was further perturbing by the magnitude of $p$ with its horizontal direction randomly chosen. Then, the particle was released to fall down and stick onto the bottom of the cell. The initial position of the second particle was randomly chosen and perturbed by the same way. Afterward, it was released from the stop end of the cell and and allowed to fall. The second particle could fall to the cell bottom or hit the first particle at an angle of attack $(\theta)$ (Fig. 1b). The second particle would stick to the first one when $\theta<\theta_{\mathrm{c}}$. Otherwise, the second particle would slide around the first particle and fall down to the bottom of the cell. The process was repeated until 2000 particles were released and a packed bed was formed. The packing characteristics of particles were clearly controlled by the parameters $p$ and $\theta_{\mathrm{c}}$. With $\theta_{\mathrm{c}}=90^{\circ}$, the falling particle, once it hits the other ones, would stick. With $\theta_{\mathrm{c}}=0^{\circ}$, the falling particle has to move down to a place contacting with the other three existing particles to attain the mechanical balance.
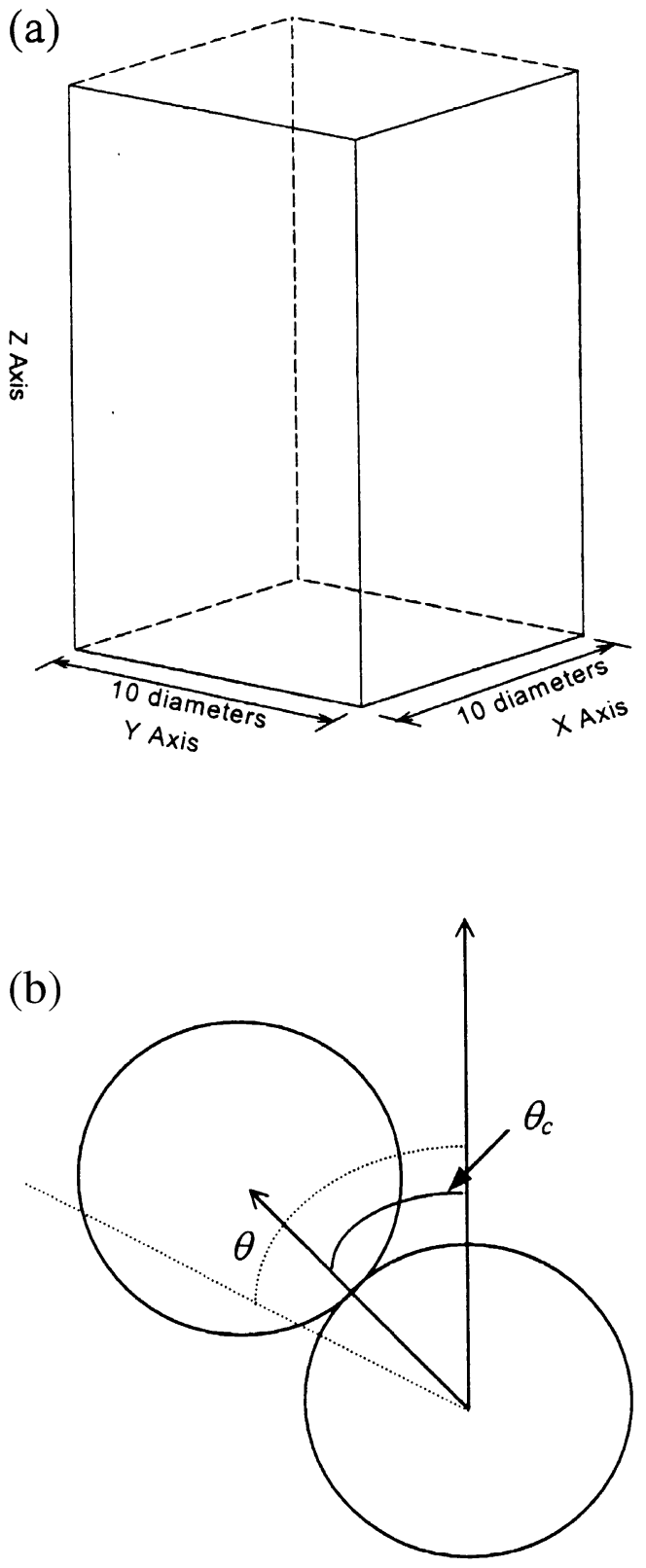

Fig. 1. (a) Schematic of the packing cell. (b) The measure of angle of attack $(\theta)$ and the critical angle $\left(\theta_{c}\right)$.

The Voronoi analysis was conducted by the method proposed by Tanemura et al. [31], from which the normalized probability distributions of the number of faces, area $(A)$, volume $(V)$, asphericity parameter $(\eta)$, and the associated Boltzmann-Gibbs entropy $(S)$ for these distributions of VP were calculated. Other details could be found in Ref. [25]. Notably, the structure of the packed bed is not homogenous in the $z$ direction, but is bound at the cell bottom and open at the top. In the present study, the packed bed of height 20-40 particle diameters was generated, depending on the value of the critical angle. To simulate a very large packed bed and eliminate the boundary effects, we chose a window for VP analysis of the 
height of 10 particle diameters. It was noted that if only the edges of the window were at least 2 particle diameters in distance away from the cell bottom and from the top surface, the VP analysis yielded the same results regardless of the choice of the window.

\section{Results and discussion}

Fig. 2 demonstrates the packed bed generated at $\theta_{c}=0^{\circ}$ or $90^{\circ}$ and $p=0.001$ or 0.2 , respectively. Apparently, the particles would be packed in an orderly manner when both $\theta_{c}$ and $p$ values are low. The corresponding bed height had also become lesser.

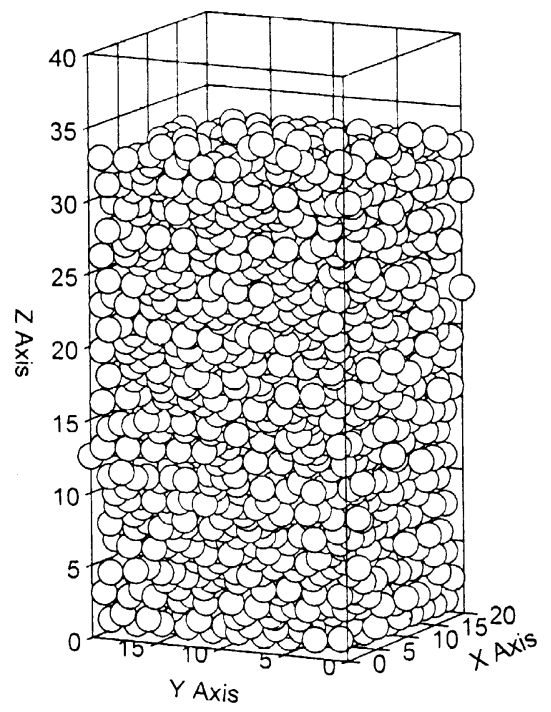

(a)

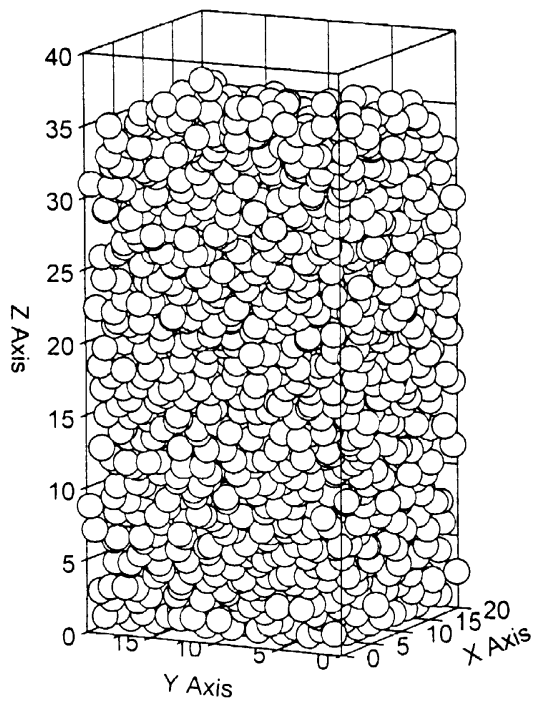

(c)
Fig. 3a and $\mathrm{b}$ illustrate the RDFs for the packed beds with $\theta_{\mathrm{c}}=0^{\circ}$ and $90^{\circ}$, respectively. Notably, at $\theta_{\mathrm{c}}=0^{\circ}$, the peaks of $g(r)$ appeared at the distances of one $(r=2)$ and of two diameters $(r=4)$, indicating the perfect center-body crystal structure for hard spheres contacting with each other. At the greater $p$, although some noises were introduced, the bed structures closely correspond to a bcc lattice.

Different characteristics were noticeable for the case with the critical angle of $90^{\circ}$. At $p<0.04$, the packing structure clearly revealed a crystal-like characteristic. With increasing $p$ value, despite the preservation of the $r=2$ peak (indicating the direct particle-particle contact), the $g(r)$ becomes flat in shape. Such an occurrence indicated

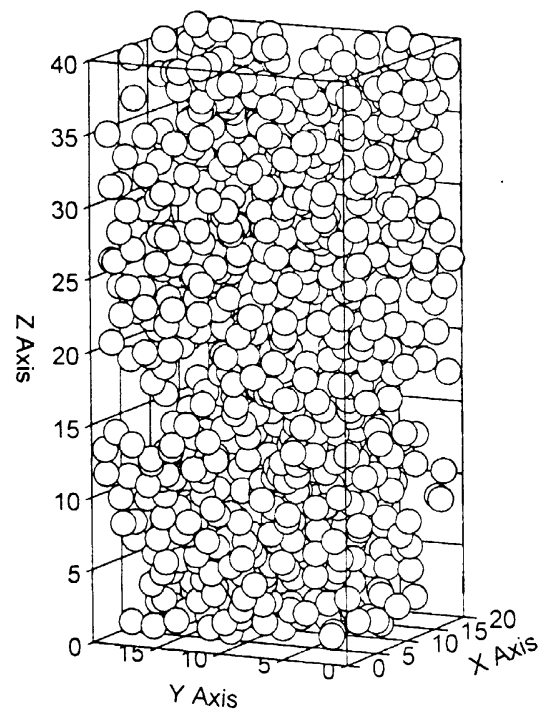

(b)

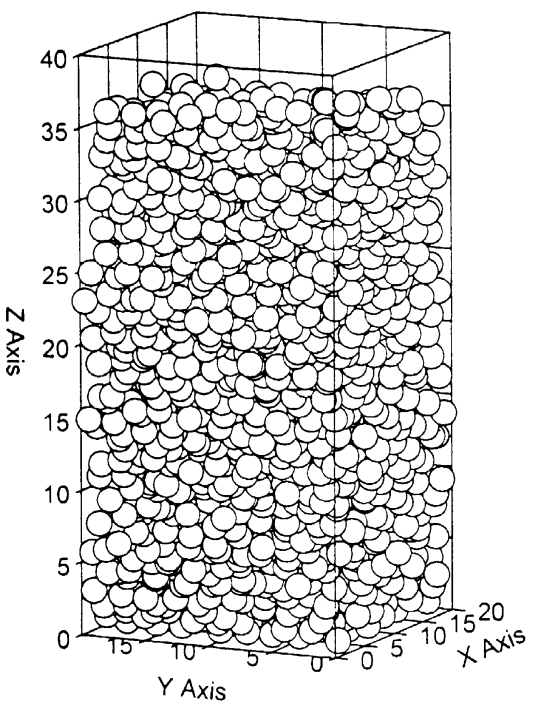

(d)

Fig. 2. Packed bed generated at (a) $\theta_{\mathrm{c}}=0^{\circ}$ and $p=0.001$, (b) $\theta_{\mathrm{c}}=90^{\circ}$ and $p=0.1$, (c) $\theta_{\mathrm{c}}=0^{\circ}$ and $p=1.0$, and (d) $\theta_{\mathrm{c}}=90^{\circ}$ and $p=0.001$. 
that the packing characteristics for the system at greater $p$ would reveal a liquid-like configuration. The occurrence of 'phase transition' among crystal-like and liquid-like structures is, hence, noticeable. Since all particles have to contact with each other, their configuration cannot reach a gas-like state as the constant-volume system [25].

At $\theta_{\mathrm{c}}=0^{\circ}$, since all particles would not stick to the existing particles but have to fall down to the position satisfying static balance of forces, the formed cake would resemble a centered body structure, thereby having a solid porosity close to 0.6 . As $\theta_{c}$ increases, the particles tend to stick to the originally existing particles in the packed bed, hence, yielding a loose bed. Such a tendency becomes more apparent when the $p$ value becomes large. At $p=$ 1.0 , the solid fraction would be reduced to 0.15 at $\theta_{\mathrm{c}}=90^{\circ}$ (data not shown).

(a)
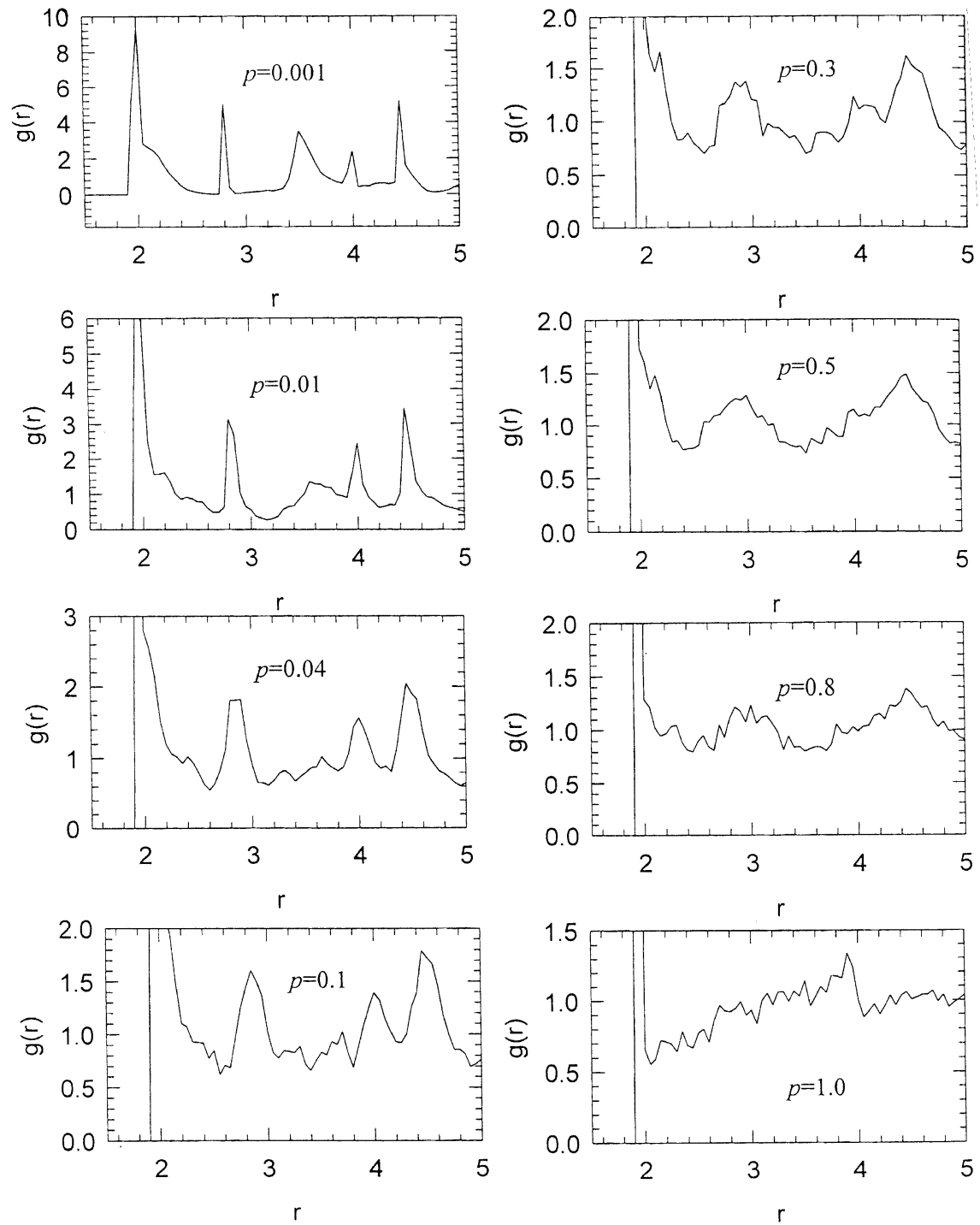

Fig. 3. Radial distribution functions (RDFs) for the packed beds with (a) $\theta_{\mathrm{c}}=0^{\circ}$ and (b) $\theta_{\mathrm{c}}=90^{\circ}$. Left to right and up to down: $p=0.001,0.01,0.04,0.1$, $0.3,0.5,0.8$, and 1.0 , respectively. 
(b)
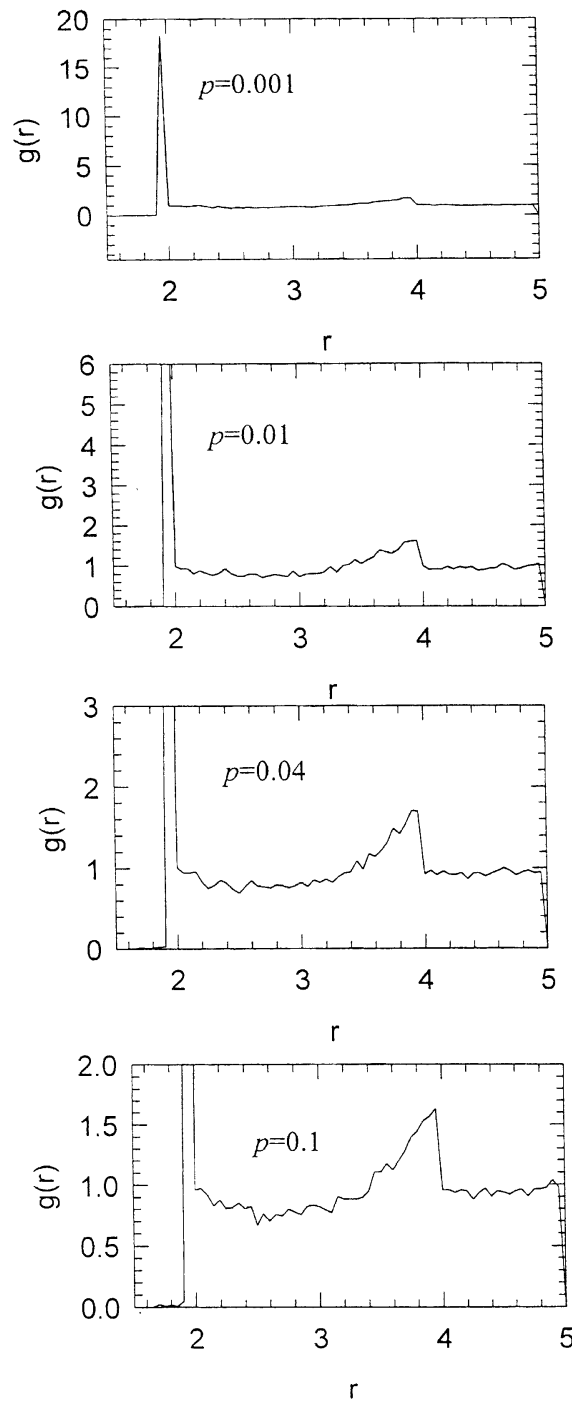
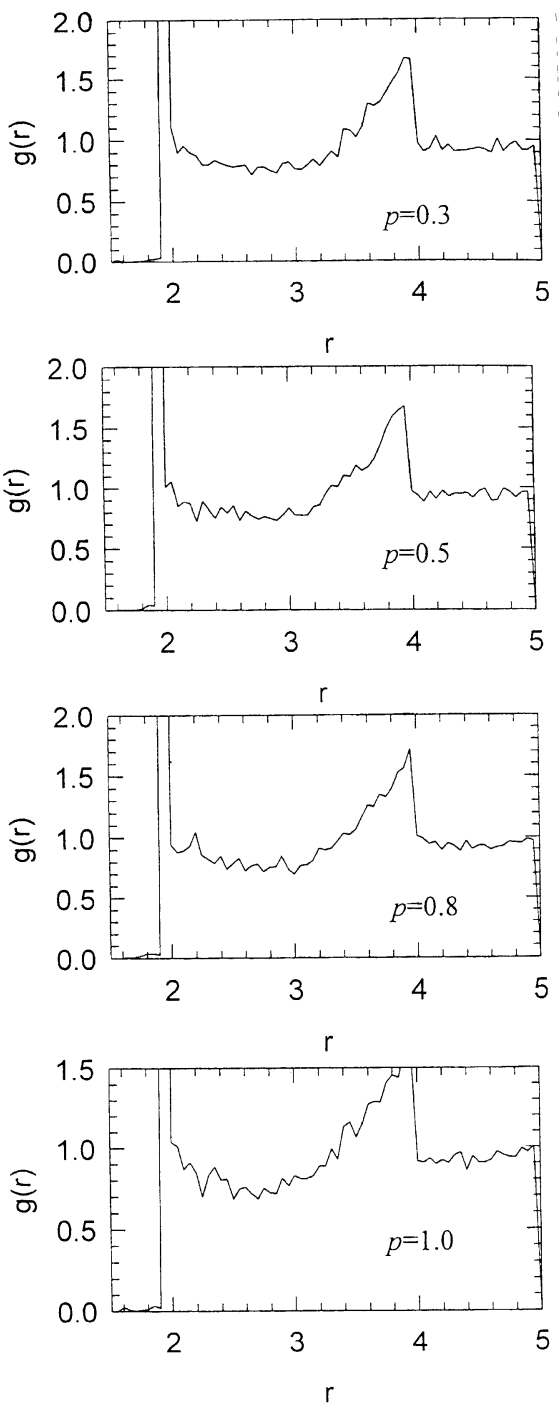

Fig. 3 (continued).

where $f$ is the probability distribution of some measure $\phi$ and the last term counts the bias for numerical partition, the analogy with phase change phenomena is established [25]. Restated, the entropy $S_{\phi}$ provides a single-value measure to the variance of a distribution. $S_{\phi}=0$ for a delta distribution and $S_{\phi}>0$ for a widely distributed function.

Fig. 5 demonstrates the configurational entropy function of VP volume $\left(S_{V}\right)$. All the entropy functions for the other microstructural descriptors resemble the shape demonstrated in Fig. 5 and are not shown here for brevity. It is worth noting that the fluctuations noted in $S_{V}$ (Fig. 5) are more easily detectable than those in $V_{\mathrm{AVE}}$ plot (Fig. 4). Hence, the former is a more sensitive microstructural descriptor to the packed bed than is the latter.

At $\theta_{\mathrm{c}}=0^{\circ}$, since the simulated packed bed would be in the form of bcc lattice, $S_{V}=0$ regardless of the magnitude of $p$. At a fixed $\theta_{c}$, marked increase in $S_{V}$ occurs when parameter $p$ increases to exceed certain threshold value. Such a marked increase corresponds to the occurrence of phase transition detected in the RDF plots (Fig. 3a and b). Restated, the increase of the configurational entropy identifies the occurrence of phase transition of packing characteristics in a packed bed.

Fig. 6a demonstrates the mean values of the calculated asphericity distributions $\left(\eta_{\mathrm{AVE}}\right)$ as the function of $\theta_{\mathrm{c}}$ and $p$ values. The corresponding variances of $\eta$ distributions become the largest at $\theta_{c}=90^{\circ}$, which are illustrated in Fig. 6b. Fig. 6c demonstrates the $\eta$ distributions at $\theta_{\mathrm{c}}=90^{\circ}$ and under various $p$. Note that since the particles were randomly perturbed, these figures contain fluctuations. At $\theta_{\mathrm{c}}=0^{\circ}$ and $/$ or $p=0.001, \eta_{\mathrm{AVE}}$ values are all around 1.35-1.40 (Fig. 6a), together with very small variance 


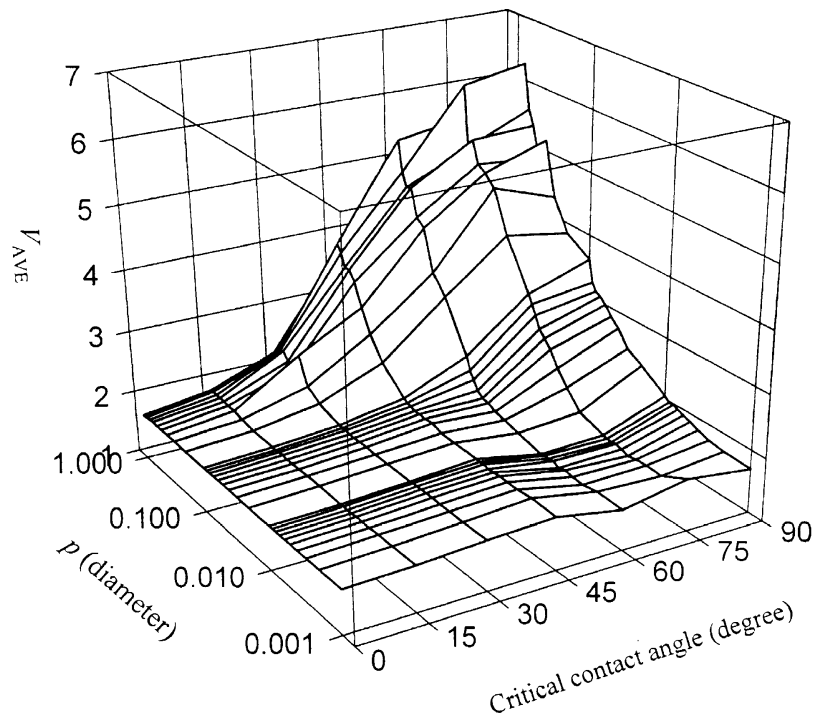

Fig. 4. Mean volume of VP of the packed bed.

(Fig. 6b), indicating that the VP in the packed bed all exhibit a shape resembling the body-centered cubic lattice. Such an observation closely corresponds to the crystal-like structure as demonstrated in RDF functions (Fig. 3a and b). At greater $\theta_{\mathrm{c}}$ and $p$ values, $\eta_{\mathrm{AVE}}$ value increases to around 1.9 with an increasing variance, indicating that the $\eta$ distribution has shifted to greater value region with a broader distribution. For example, as Fig. $6 \mathrm{c}$ depicts, the $\eta$ distribution covers a wide range from 1.3 to 2.3 at $\theta_{\mathrm{c}}=90^{\circ}$ and $p=1.0$. Restated, the corresponding VP have a variety of shapes, ranging from that resembling body-centered

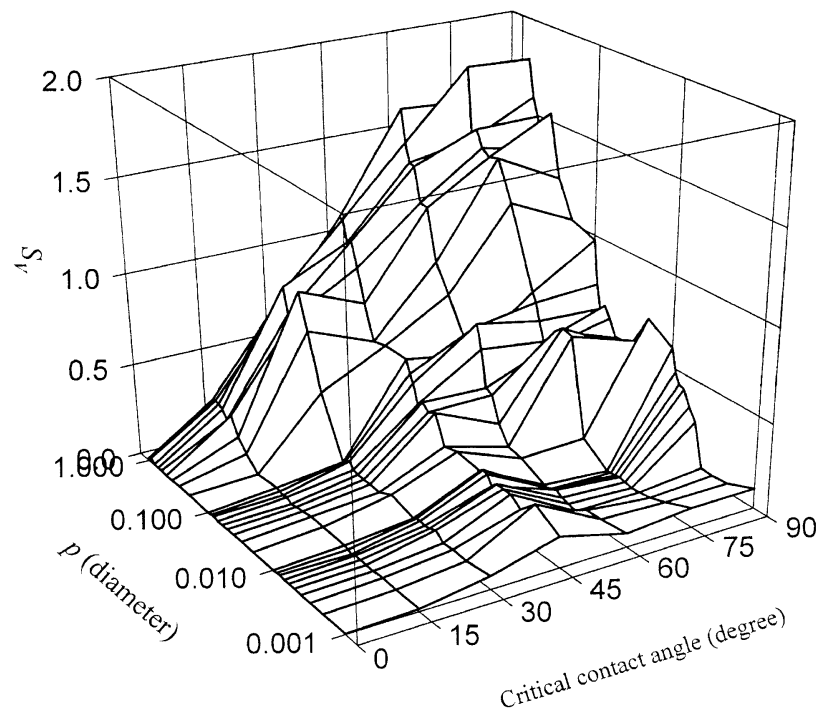

Fig. 5. Configurational entropy function of VP volume $\left(S_{V}\right)$ of the packed bed. cubic $(\eta=1.33)$ to that among simple cubic $(\eta=1.91)$ and tetrahedron $(\eta=3.31)$ and many other elongated, disordered shapes. As Fig. 6c reveals, the variance of $\eta$ distribution starts to increase at $p=0.03 \sim 0.04$. This trend could be used as an index to identify the phase transition among crystal-like and liquid-like states for particle packing.

By using the change in mean values (VP volume or $\left.\eta_{\mathrm{AVE}}\right)$ or entropy function $\left(S_{V}\right)$ of VP characteristics (and all the other indices as well), the $p$ value at which phase change occurs could be identified. Table 1 lists the corresponding phase change points using $V_{\mathrm{AVE}}, \eta_{\mathrm{AVE}}$, and $S_{V}$ as the indicators. As Table 1 reveals, using $V_{\mathrm{AVE}}$ as an indicator the phase change could be detected at $\theta_{\mathrm{c}}>15^{\circ}$. The other two indicators are apparently more sensitive than the mean volume is. Moreover, the $p$ value required including phase transition decreases with the increasing $\theta_{c}$. Restated, if the particle-particle interaction has become
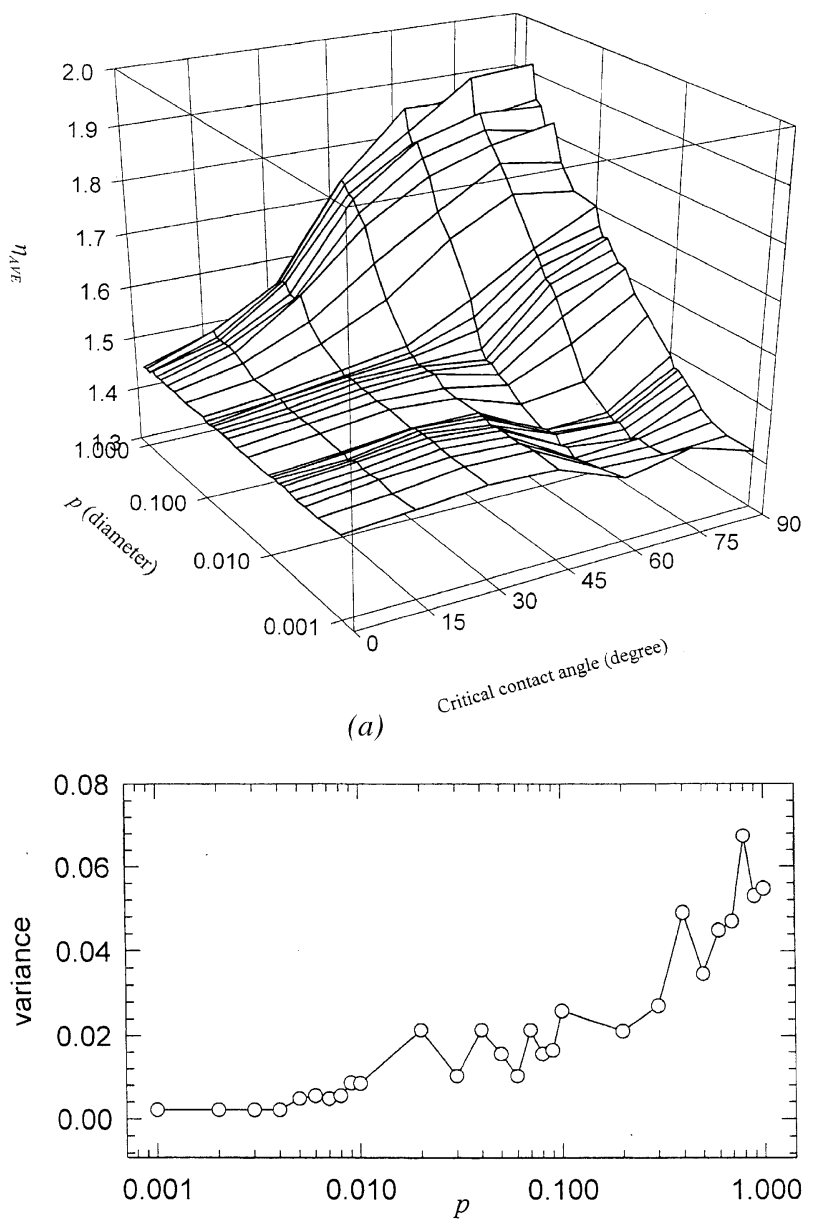

(b)

Fig. 6. Asphericity of VP of the packed bed. (a) $\eta_{\mathrm{AVE}}$; (b) variance of $\eta$ distributions at $\theta_{\mathrm{c}}=90^{\circ}$; and (c) the $\eta$ distributions at $\theta_{\mathrm{c}}=90^{\circ}$. Left to right and up to down: $p=0.001,0.01,0.04,0.1,0.3,0.5,0.8$, and 1.0, respectively. 
(c)
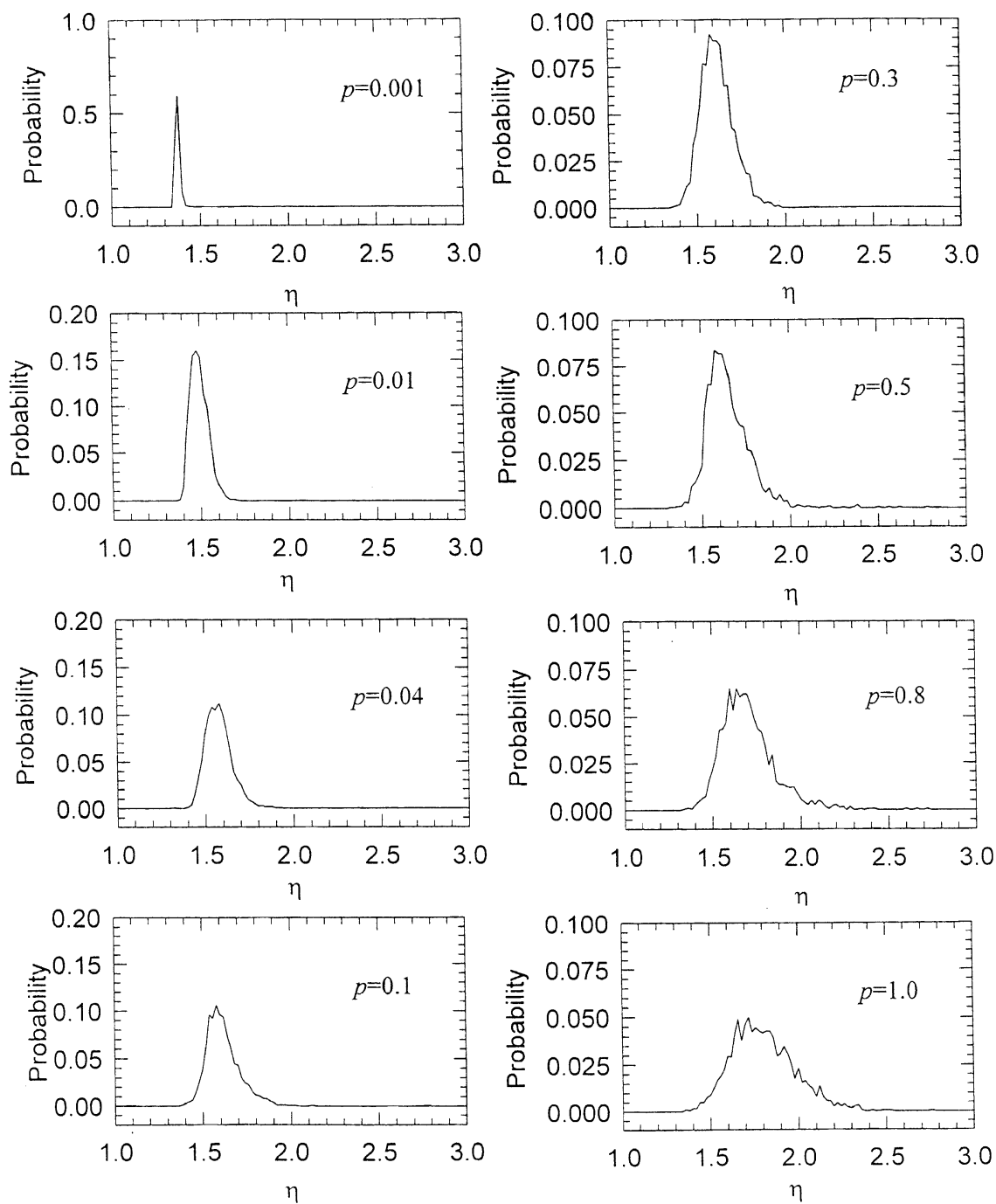

Fig. 6 (continued)

strong enough (larger $\theta_{\mathrm{c}}$ ), the phase transition could more readily occur at a small perturbation of the initial position of particles.

Table 1

The $p$ values where solid-liquid phase change occurs at various critical contact angles using $V_{\mathrm{AVE}}, S_{V}$, and $\eta_{\mathrm{AVE}}$ values

\begin{tabular}{llll}
\hline $\begin{array}{l}\text { Critical } \\
\text { contact } \\
\text { angle }\left({ }^{\circ}\right)\end{array}$ & $\begin{array}{l}\text { Range of } p \\
\text { value }\left(V_{\mathrm{AVE}}\right)\end{array}$ & $\begin{array}{l}\text { Range of } p \\
\text { value }\left(S_{V}\right)\end{array}$ & $\begin{array}{l}\text { Range of } p \\
\text { value }\left(\eta_{\mathrm{AVE}}\right)\end{array}$ \\
\hline 0 & $\mathrm{NA}^{\mathrm{a}}$ & $\mathrm{NA}$ & $\mathrm{NA}$ \\
15 & $\mathrm{NA}$ & $0.2-0.3$ & $0.2-0.3$ \\
30 & $0.2-0.3$ & $0.1-0.2$ & $0.1-0.2$ \\
45 & $0.1-0.2$ & $0.04-0.05$ & $0.09-0.1$ \\
60 & $0.07-0.08$ & $0.03-0.04$ & $0.05-0.06$ \\
75 & $0.03-0.04$ & $0.01-0.02$ & $0.02-0.03$ \\
90 & $0.006-0.007$ & $0.004-0.005$ & $0.005-0.006$ \\
\hline
\end{tabular}

${ }^{\mathrm{a}}$ Not available.

\section{Conclusions}

We examined the feasibility of using Voronoi polyhedron (VP) for describing the microstructures of a simulated packed bed with controlled disorderliness using the critical angle $\left(\theta_{c}\right)$ and perturbed distance $(p)$ as parameters. The RDFs revealed that the microstructure of packed bed transited from a regularly packed (crystal-like) to a loosely packed (liquid-like) pattern when both the $\theta_{c}$ and $p$ had become large. In conjunction with the occurrence of this transition, the normalized probability distributions of the number of faces, area $(A)$, volume $(V)$, asphericity parameter $(\eta)$, and the associated Boltzmann-Gibbs entropy $(S)$ for these distributions of VP changed accordingly.

VP could provide more configurational information regarding the packing characteristics in the packed bed. Among all VP descriptors investigated, the mean asphericity and the entropy function of VP volume were noted 
adequate indices for packing characteristics of the packed bed. The resulting asphericity distribution illustrated that all VP exhibit a shape resembling the body-centered cubic lattice in solid-like configuration. After phase transition, the VP have a variety of shapes, ranging from that resembling body-centered cubic to that among simple cubic and tetrahedron and many other elongated, disordered shapes. The critical $p$ values corresponding to the occurrence of solid/liquid transition in microstructure of packed bed were identified and tabulated.

\section{List of symbols}

A surface area of VP $\left(\mathrm{m}^{2}\right)$

$f \quad$ distribution of measure $(-)$

$g \quad$ radial distribution function $(-)$

$p \quad$ degree of disorderliness $(-)$

$r \quad$ radial direction $(\mathrm{m})$

$S_{V} \quad$ entropy for VP volume (-)

$S_{\phi} \quad$ entropy for measure, $\phi(-)$

$V \quad$ volume of VP $\left(\mathrm{m}^{3}\right)$

$V_{\mathrm{AVE}} \quad$ average volume of $\mathrm{VP}\left(\mathrm{m}^{3}\right)$

\section{Greek letters}

$\Psi \quad$ probability density function of the physical quantity $b(-)$

$\theta \quad$ angle of attack $\left({ }^{\circ}\right)$

$\theta_{c} \quad$ critical contact angle $\left({ }^{\circ}\right)$

$\eta \quad$ asphericity of VP (-)

$\eta_{\mathrm{AVE}} \quad$ average asphericity of VP (-)

$\phi \quad$ measure of some distributions (-)

\section{Acknowledgements}

The authors appreciate Prof. C.Y. Mou of Department of Chemistry, National Taiwan University, for providing the VP program.

\section{References}

[1] G.F. Voronoi, J. Reine, Angew. Math. 134 (1908) 198.

[2] J.D. Bernal, Proc. R. Soc. London, Ser. A 280 (1964) 299.

[3] J.D. Bernal, J.L. Finney, Discuss. Faraday Soc. 43 (1967) 62.

[4] I.L. Finney, Proc. R. Soc. London, Ser. A 319 (1970) 495.

[5] M.R. Hoare, J. Non-Cryst. Solids 31 (1978) 157.

[6] H.C. Hsu, A. Rahman, J. Chem. Phys. 71 (1979) 4979.

[7] G. Ruocco, M. Sampoli, R. Vallauri, J. Mol. Struct. 250 (1991) 259.

[8] T.J. Hsu, C.Y. Mou, Mol. Phys. 75 (1992) 1329.

[9] J.C.G. Motoro, J.L.F. Abascal, J. Chem. Phys. 97 (1993) 4211.

[10] J.C.G. Motoro, F. Bresme, J.L.F. Abascal, J. Chem. Phys. 101 (1994) 10892.

[11] J.P. Shih, S.Y. Sheu, C.Y. Mou, J. Chem. Phys. 100 (1994) 2202.

[12] M.I. Aoki, K. Tsumuraya, J. Chem. Phys. 104 (1996) 6719.

[13] P. Jund, D. Caprion, R. Jullien, Europhys. Lett. 37 (1997) 547.

[14] A. Baranyai, I. Ruff, J. Chem. Phys. 85 (1986) 365.

[15] A. Goede, R. Preissner, C. Frommel, J. Comput. Chem. 18 (1997) 1113.

[16] J. Bohm, M. Bohm, R.B. Heimann, Cryst. Res. Technol. 31 (1996) 1069.

[17] N.W. Thomas, Acta Crystallogr., Sect. B: Struct. Sci. 52 (1996) 939.

[18] S. Ghosh, Z. Nowak, K. Lee, Acta Mater. 45 (1997) 2215.

[19] S. Moorthy, S. Ghosh, Comput. Methods Appl. Mech. Eng. 151 (1998) 377.

[20] R.E. Eils, K. Bertin, B. Saracoglu, E. Rinke, F. Schrock, Y. Parazza, M. Usson, Z.H.K. Robertnicoud, J.M. Stelzer, T. Chassery, C. Cremer, J. Microsc. 177 (1995) 150.

[21] M. Gerstein, J. Tsai, M. Levitt, J. Mol. Biol. 249 (1995) 955.

[22] J.D. Rogers, R.L. Long, J. Membr. Sci. 134 (1997) 1.

[23] D.H. Everett, Discuss. Faraday Soc. 43 (1967) 82.

[24] Y.C. Liao, D.J. Lee, Chem. Eng. Commun. 176 (1999) 77.

[25] Y.C. Liao, D.J. Lee, B.H. Chen, Powder Technol., in press.

[26] G.J. Cheng, A.B. Yu, P. Zulli, Chem. Eng. Sci. 54 (1999) 4199.

[27] M. Tassopoulos, D.E. Rosner, AIChE J. 38 (1992) 15.

[28] P.L. Spedding, R.M. Spencer, Comput. Chem. Eng. 22 (1998) 247.

[29] W.M. Lu, K.J. Huang, AIChE J. 41 (1995) 1443.

[30] M. Suzuki, T. Oshima, Powder Technol. 36 (1983) 181.

[31] M. Tanemura, T. Ogawa, N. Ogita, J. Comput. Phys. 51 (1983) 191. 